\title{
On the Fibonacci quaternion sequence with quadruple-produce components
}

\author{
Orhan Dişkaya and Hamza Menken
}

\begin{abstract}
This paper examines the Fibonacci quaternion sequence with quadruple-produce components, and demonstrates a golden-like ratio and some identities for this sequence. Its generating and exponential generating functions are given. Along with these, its series and binomial sum formula are established.
\end{abstract}

\section{Introduction}

Quaternions were examined by William Rowan Hamilton (1805-1865) as an extension of the complex numbers. There has been an increasing interest in algebra problems on quaternion field since many algebra problems on the quaternion field were encountered in some of the applied sciences, such as differential geometry, quantum physics, geostatics, and analysis [6]. A quaternion is a hyper-complex number. As usual, let $\mathbb{C}$ and $\mathbb{R}$ denote the fields of the complex and real numbers, respectively. Let $\mathbf{Q}$ be a fourdimensional vector space over $\mathbb{R}$ with an ordered basis, denoted by $1, i, j, k$. A quaternion is defined by the following equation:

$$
q=q_{0}+i q_{1}+j q_{2}+k q_{3},
$$

where $q_{0}, q_{1}, q_{2}$ and $q_{3}$ are real numbers [17].

In $[3,11]$, the quaternion multiplication is defined by the rules

$$
\begin{gathered}
1^{2}=1, \quad i^{2}=j^{2}=k^{2}=-1 \\
i j=-j i=k, \quad j k=-k j=i, \quad k i=-i k=j .
\end{gathered}
$$

Multiplication of quaternions is associative and non-commutative.

Received March 18, 2021.

2020 Mathematics Subject Classification. 11B39, 11B83, 05A15.

Key words and phrases. Fibonacci numbers, golden ratio, quaternion, generating function, exponential generating function, series, binomial sum.

https://doi.org/10.12697/ACUTM.2021.25.11

Corresponding author: Hamza Menken 
Let $q=q_{0}+i q_{1}+j q_{2}+k q_{3}$ and $p=p_{0}+i p_{1}+j p_{2}+k p_{3}$ be any two quaternions. Then the addition and subtraction of them are

$$
q \mp p=\left(q_{0} \mp p_{0}\right)+i\left(q_{1} \mp p_{1}\right)+j\left(q_{2} \mp p_{2}\right)+k\left(q_{3} \mp p_{3}\right),
$$

and multiplication of them is

$$
\begin{aligned}
q p= & \left(q_{0} p_{0}-q_{1} p_{1}-q_{2} p_{2}-q_{3} p_{3}\right)+i\left(q_{0} p_{1}+q_{1} p_{0}+q_{2} p_{3}-q_{3} p_{2}\right) \\
& +j\left(q_{0} p_{2}+q_{2} p_{0}+q_{3} p_{1}-q_{1} p_{3}\right)+k\left(q_{0} p_{3}+q_{3} p_{0}+q_{1} p_{2}-q_{2} p_{1}\right) .
\end{aligned}
$$

The conjugate and norm of a quaternion are

$$
\bar{q}=q_{0}-i q_{1}-j q_{2}-k q_{3}
$$

and

$$
N(q)=q \bar{q}=q_{0}^{2}+q_{1}^{2}+q_{2}^{2}+q_{3}^{2} .
$$

More information about the quaternions can be found in $[1,6,10,17]$.

Horadam [8] described the $n$th Fibonacci quaternion as

$$
Q_{n}=F_{n}+i F_{n+1}+j F_{n+2}+k F_{n+3},
$$

where $F_{n}$ represents the $n$th Fibonacci number.

In [5] a non-homogeneous third-order recursive sequence is studied as a generalization of Fibonacci numbers and various identities are given. In [4] the authors introduce and study the Lucas generalized incomplete Fibonacci polynomials and give some properties of them. In [6] the Fibonacci quaternions are studied. In [15] the $q$-analogues of the incomplete Fibonacci and Lucas polynomials are studied and some properties are given. In [13] the authors define an upper bound of the third Hankel determinant for a subclass of $q$-starlike functions associated with $k$-Fibonacci numbers. In the survey article [14] the importance and widespread applications of the $q$ - in analysis are given in detail.

In the present work we define the Fibonacci quaternion sequence with quadruple-produce components, and we investigate its various properties.

\section{The Fibonacci quaternion sequence with quadruple-produce components}

The number expressed by $Q(a, b, c, d)$ is defined at the set of quaternion integers $(a, b, c, d)=a+i b+j c+k d$, where $a, b, c, d \in \mathbb{Z}$. The Fibonacci sequence $\left\{F_{n}\right\}_{n \geq 0}$ is defined by

$$
F_{0}=0, \quad F_{1}=1, \quad \text { and } \quad F_{n+2}=F_{n+1}+F_{n}
$$

for all $n \geq 0$. Here, $F_{n}$ is the $n$th Fibonacci number $[4,15,13]$. First few terms of this sequence are $1,1,2,3,5,8,13,21,34,55,89,144$. The 
recurrence involves the characteristic equation $x^{2}-x-1=0$. The roots of this equation are

$$
\alpha=(1+\sqrt{5}) / 2 \text { and } \beta=(1-\sqrt{5}) / 2 .
$$

From this we derive that $\alpha+\beta=1, \quad \alpha-\beta=\sqrt{5}$, and $\alpha \beta=-1$. The conclusions of [12] reveal that the following identities hold:

$$
\begin{aligned}
\lim _{n \rightarrow \infty} \frac{F_{n+1}}{F_{n}} & =\phi \quad(\text { Golden ratio) }, \\
\frac{\alpha^{n}-\beta^{n}}{\alpha-\beta} & =F_{n} \quad \text { (Binet formula) } \\
\sum_{n=0}^{m} F_{n} & =F_{m+2}-1 \quad \text { (Fibonacci sum) } \\
\sum_{n=0}^{\infty} F_{n} x^{n} & =\frac{x}{1-x-x^{2}} \quad \text { (Generating function) }, \\
\sum_{n=0}^{\infty} \frac{F_{n}}{n !} x^{n} & =\frac{e^{\alpha x}-e^{\beta x}}{\alpha-\beta} \quad \text { (Exp. generating function), } \\
\sum_{n=0}^{\infty} \frac{F_{n}}{t^{n+1}} & =\frac{1}{t^{2}-t-1} \quad \text { (Fibonacci series), } \\
\sum_{n=0}^{m}\left(\begin{array}{c}
m \\
n
\end{array}\right) F_{n} & =F_{2 m} \quad \text { (Binomial sum). }
\end{aligned}
$$

In $[2,7,9,10,16]$, the number $Q(a, b, c, d)$ is defined by the four-dimensional recurrences

$$
\begin{aligned}
& Q(a+2, b, c, d)=Q(a+1, b, c, d)+Q(a, b, c, d), \\
& Q(a, b+2, c, d)=Q(a, b+1, c, d)+Q(a, b, c, d), \\
& Q(a, b, c+2, d)=Q(a, b, c+1, d)+Q(a, b, c, d), \\
& Q(a, b, c, d+2)=Q(a, b, c, d+1)+Q(a, b, c, d),
\end{aligned}
$$

where

$$
\begin{gathered}
Q(0,0,0,0)=0, \quad Q(1,0,0,0)=1, \quad Q(0,1,0,0)=i, \\
Q(0,0,1,0)=j, \quad Q(0,0,0,1)=k, \quad Q(1,1,0,0)=1+i, \\
Q(1,0,1,0)=1+j, \quad Q(1,0,0,1)=1+k, \\
Q(1,1,1,0)=1+i+j, \quad Q(1,1,0,1)=1+i+k, \\
Q(1,0,1,1)=1+j+k, \quad Q(0,1,1,0)=i+j, \\
Q(0,1,0,1)=i+k, \quad Q(0,0,1,1)=j+k, \\
Q(0,1,1,1)=i+j+k, \quad Q(1,1,1,1)=1+i+j+k .
\end{gathered}
$$


Therefore, the number $Q(a, b, c, d)$ has a Fibonacci recurrence for each coordinate. From (10), the case $b=c=d=0$ gives

$$
Q(a+2,0,0,0)=Q(a+1,0,0,0)+Q(a, 0,0,0),
$$

and by the initial conditions $Q(0,0,0,0)=0$ and $Q(1,0,0,0)=1$, we obtain

$$
Q(a, 0,0,0)=F_{a} .
$$

The case $b=1$ and $c=d=0$ gives

$$
Q(a+2,1,0,0)=Q(a+1,1,0,0)+Q(a, 1,0,0),
$$

and with the initial conditions $Q(0,1,0,0)=i$ and $Q(1,1,0,0)=1+i$ we obtain

$$
Q(a, 1,0,0)=F_{a}+i F_{a+1} .
$$

The case $c=1$ and $b=d=0$ gives

$$
Q(a+2,0,1,0)=Q(a+1,0,1,0)+Q(a, 0,1,0),
$$

and with the initial conditions $Q(0,0,1,0)=j$ and $Q(1,0,1,0)=1+j$ we obtain

$$
Q(a, 0,1,0)=F_{a}+j F_{a+1} .
$$

The case $d=1$ and $b=c=0$ gives

$$
Q(a+2,0,0,1)=Q(a+1,0,0,1)+Q(a, 0,0,1),
$$

and with the initial conditions $Q(0,0,0,1)=k$ and $Q(1,0,0,1)=1+k$ we obtain

$$
Q(a, 0,0,1)=F_{a}+k F_{a+1} .
$$

The case $b=c=1$ and $d=0$ gives

$$
Q(a+2,1,1,0)=Q(a+1,1,1,0)+Q(a, 1,1,0),
$$

and with the initial conditions $Q(0,1,1,0)=i+j$ and $Q(1,1,1,0)=1+i+j$ we obtain

$$
Q(a, 1,1,0)=F_{a}+(i+j) F_{a+1} .
$$

The case $b=d=1$ and $c=0$ gives

$$
Q(a+2,1,0,1)=Q(a+1,1,0,1)+Q(a, 1,0,1),
$$

and with the initial conditions $Q(0,1,0,1)=i+k$ and $Q(1,1,0,1)=1+i+k$ we obtain

$$
Q(a, 1,0,1)=F_{a}+(i+k) F_{a+1} .
$$

The case $c=d=1$ and $b=0$ gives

$$
Q(a+2,0,1,1)=Q(a+1,0,1,1)+Q(a, 0,1,1),
$$

and with the initial conditions $Q(0,0,1,1)=j+k$ and $Q(1,0,1,1)=1+j+k$ we obtain

$$
Q(a, 0,1,1)=F_{a}+(j+k) F_{a+1} .
$$


The case $b=c=d=1$ gives

$$
Q(a+2,1,1,1)=Q(a+1,1,1,1)+Q(a, 1,1,1),
$$

and with the initial conditions $Q(0,1,1,1)=i+j+k$ and $Q(1,1,1,1)=$ $1+i+j+k$ we obtain

$$
Q(a, 1,1,1)=F_{a}+(i+j+k) F_{a+1} .
$$

In the same way, from the recurrence (11) we have

$$
\begin{aligned}
& Q(0, b, 0,0)=i F_{b}, \\
& Q(1, b, 0,0)=i F_{b}+F_{b+1}, \\
& Q(0, b, 1,0)=i F_{b}+j F_{b+1}, \\
& Q(0, b, 0,1)=i F_{b}+k F_{b+1}, \\
& Q(1, b, 1,0)=i F_{b}+(1+j) F_{b+1}, \\
& Q(1, b, 0,1)=i F_{b}+(1+k) F_{b+1}, \\
& Q(0, b, 1,1)=i F_{b}+(j+k) F_{b+1}, \\
& Q(1, b, 1,1)=i F_{b}+(1+j+k) F_{b+1} .
\end{aligned}
$$

From the recurrence (12) we also get

$$
\begin{aligned}
& Q(0,0, c, 0)=j F_{c}, \\
& Q(1,0, c, 0)=j F_{c}+F_{c+1}, \\
& Q(0,1, c, 0)=j F_{c}+i F_{c+1}, \\
& Q(0,0, c, 1)=j F_{c}+k F_{c+1}, \\
& Q(1,1, c, 0)=j F_{c}+(1+i) F_{c+1}, \\
& Q(1,0, c, 1)=j F_{c}+(1+k) F_{c+1}, \\
& Q(0,1, c, 1)=j F_{c}+(i+k) F_{c+1}, \\
& Q(1,1, c, 1)=j F_{c}+(1+i+k) F_{c+1} .
\end{aligned}
$$

From the recurrence (13) we get

$$
\begin{aligned}
& Q(0,0,0, d)=k F_{d}, \\
& Q(1,0,0, d)=k F_{d}+F_{d+1}, \\
& Q(0,1,0, d)=k F_{d}+i F_{d+1}, \\
& Q(0,0,1, d)=k F_{d}+j F_{d+1}, \\
& Q(1,1,0, d)=k F_{d}+(1+i) F_{d+1}, \\
& Q(1,0,1, d)=k F_{d}+(1+j) F_{d+1}, \\
& Q(0,1,1, d)=k F_{d}+(i+j) F_{d+1}, \\
& Q(1,1,1, d)=k F_{d}+(1+i+j) F_{d+1} .
\end{aligned}
$$


Considering the method of Harman [7], the recurrence (11), and the initial conditions $Q(a, 0,0,0)=F_{a}$ and $Q(a, 1,0,0)=F_{a}+i F_{a+1}$, it follows that

$$
\begin{aligned}
Q(a, b, 0,0) & =F_{b-1} Q(a, 0,0,0)+F_{b} Q(a, 1,0,0) \\
& =F_{b-1} F_{a}+F_{b}\left(F_{a}+i F_{a+1}\right)=\left(F_{b-1}+F_{b}\right) F_{a}+i F_{b} F_{a+1} \\
& =F_{b+1} F_{a}+i F_{b} F_{a+1} .
\end{aligned}
$$

From (11), together with the initial conditions $Q(a, 0,1,0)=F_{a}+j F_{a+1}$ and $Q(a, 1,1,0)=F_{a}+(i+j) F_{a+1}$, it follows that

$$
\begin{aligned}
Q(a, b, 1,0) & =F_{b-1} Q(a, 0,1,0)+F_{b} Q(a, 1,1,0) \\
& =F_{b-1}\left(F_{a}+j F_{a+1}\right)+F_{b}\left(F_{a}+(i+j) F_{a+1}\right) \\
& =F_{b-1} F_{a}+j F_{b-1} F_{a+1}+F_{b} F_{a}+(i+j) F_{b} F_{a+1} \\
& =F_{b+1} F_{a}+i F_{b} F_{a+1}+j F_{b+1} F_{a+1} .
\end{aligned}
$$

From (11), together with the initial conditions $Q(a, 0,0,1)=F_{a}+k F_{a+1}$ and $Q(a, 1,0,1)=F_{a}+(i+k) F_{a+1}$, it follows that

$$
\begin{aligned}
Q(a, b, 0,1) & =F_{b-1} Q(a, 0,0,1)+F_{b} Q(a, 1,0,1) \\
& =F_{b-1}\left(F_{a}+k F_{a+1}\right)+F_{b}\left(F_{a}+(i+k) F_{a+1}\right) \\
& =F_{b-1} F_{a}+k F_{b-1} F_{a+1}+F_{b} F_{a}+(i+k) F_{b} F_{a+1} \\
& =F_{b+1} F_{a}+i F_{b} F_{a+1}+k F_{b+1} F_{a+1} .
\end{aligned}
$$

From (11), together with the initial conditions $Q(a, 0,1,1)=F_{a}+(j+k) F_{a+1}$ and $Q(a, 1,1,1)=F_{a}+(i+j+k) F_{a+1}$, it follows that

$$
\begin{aligned}
Q(a, b, 0,1) & =F_{b-1} Q(a, 0,0,1)+F_{b} Q(a, 1,0,1) \\
& =F_{b-1}\left(F_{a}+(j+k) F_{a+1}\right)+F_{b}\left(F_{a}+(i+j+k) F_{a+1}\right) \\
& =F_{b-1} F_{a}+(j+k) F_{b-1} F_{a+1}+F_{b} F_{a}+i F_{b} F_{a+1}+(j+k) F_{b} F_{a+1} \\
& =F_{b+1} F_{a}+i F_{b} F_{a+1}+(j+k) F_{b+1} F_{a+1} .
\end{aligned}
$$

With the recurrence $(12)$ and the initial conditions $Q(a, b, 0,0)=F_{b+1} F_{a}+$ $i F_{b} F_{a+1}$ and $Q(a, b, 1,0)=F_{b+1} F_{a}+i F_{b} F_{a+1}+j F_{b+1} F_{a+1}$, it follows that

$$
\begin{aligned}
& Q(a, b, c, 0)=F_{c-1} Q(a, b, 0,0)+F_{c} Q(a, b, 1,0) \\
& =F_{c-1}\left(F_{b+1} F_{a}+i F_{b} F_{a+1}\right)+F_{c}\left(F_{b+1} F_{a}+i F_{b} F_{a+1}+j F_{b+1} F_{a+1}\right) \\
& =F_{c-1} F_{b+1} F_{a}+i F_{c-1} F_{b} F_{a+1}+F_{c} F_{b+1} F_{a}+i F_{c} F_{b} F_{a+1}+j F_{c} F_{b+1} F_{a+1} \\
& =F_{c+1} F_{b+1} F_{a}+i F_{c+1} F_{b} F_{a+1}+j F_{c} F_{b+1} F_{a+1} .
\end{aligned}
$$

From (12), together with the initial conditions $Q(a, b, 0,1)=F_{b+1} F_{a}+$ $i F_{b} F_{a+1}+k F_{b+1} F_{a+1}$ and $Q(a, b, 1,1)=F_{b+1} F_{a}+i F_{b} F_{a+1}+(j+k) F_{b+1} F_{a+1}$, it follows that

$$
\begin{aligned}
Q(a, b, c, 1) & =F_{c-1} Q(a, b, 0,1)+F_{c} Q(a, b, 1,1) \\
& =F_{c-1}\left(F_{b+1} F_{a}+i F_{b} F_{a+1}+k F_{b+1} F_{a+1}\right)
\end{aligned}
$$




$$
\begin{aligned}
& +F_{c}\left(F_{b+1} F_{a}+i F_{b} F_{a+1}+(j+k) F_{b+1} F_{a+1}\right) \\
= & F_{c-1} F_{b+1} F_{a}+i F_{c-1} F_{b} F_{a+1}+k F_{c-1} F_{b+1} F_{a+1} \\
& +F_{c} F_{b+1} F_{a}+i F_{c} F_{b} F_{a+1}+(j+k) F_{c} F_{b+1} F_{a+1} \\
= & F_{c+1} F_{b+1} F_{a}+i F_{c+1} F_{b} F_{a+1}+j F_{c} F_{b+1} F_{a+1}+k F_{c+1} F_{b+1} F_{a+1} .
\end{aligned}
$$

It follows from the recurrence (13) and the initial conditions $Q(a, b, c, 0)=$ $F_{c+1} F_{b+1} F_{a}+i F_{c+1} F_{b} F_{a+1}+j F_{c} F_{b+1} F_{a+1}$ and $Q(a, b, c, 1)=F_{c+1} F_{b+1} F_{a}+$ $i F_{c+1} F_{b} F_{a+1}+j F_{c} F_{b+1} F_{a+1}+k F_{c+1} F_{b+1} F_{a+1}$ that

$$
\begin{aligned}
& Q(a, b, c, d)=F_{d-1} Q(a, b, c, 1)+F_{d} Q(a, b, c, 1) \\
& =F_{d-1}\left(F_{c+1} F_{b+1} F_{a}+i F_{c+1} F_{b} F_{a+1}+j F_{c} F_{b+1} F_{a+1}\right) \\
& \quad+F_{d}\left(F_{c+1} F_{b+1} F_{a}+i F_{c+1} F_{b} F_{a+1}+j F_{c} F_{b+1} F_{a+1}+k F_{c+1} F_{b+1} F_{a+1}\right) .
\end{aligned}
$$

Hence, we obtain the Fibonacci quaternion sequence with quadruple-produce components as

$$
\begin{aligned}
Q(a, b, c, d)= & F_{d+1} F_{c+1} F_{b+1} F_{a}+i F_{d+1} F_{c+1} F_{b} F_{a+1} \\
& +j F_{d+1} F_{c} F_{b+1} F_{a+1}+k F_{d} F_{c+1} F_{b+1} F_{a+1} .
\end{aligned}
$$

The conjugate of the Fibonacci quaternion sequence with quadruple-produce components is

$$
\begin{aligned}
\overline{Q(a, b, c, d)}= & F_{d+1} F_{c+1} F_{b+1} F_{a}-i F_{d+1} F_{c+1} F_{b} F_{a+1} \\
& -j F_{d+1} F_{c} F_{b+1} F_{a+1}-k F_{d} F_{c+1} F_{b+1} F_{a+1} .
\end{aligned}
$$

Some numerical examples of $Q(a, b, c, d)$ are

$$
\begin{aligned}
& Q(0,0,0,0)=0, \\
& Q(1,1,1,1)=1+i+j+k, \\
& Q(2,2,2,2)=8+8 i+8 j+8 k, \\
& Q(2,0,1,3)=3+6 j+4 k, \\
& Q(1,2,3,4)=30+15 i+20 j+18 k .
\end{aligned}
$$

If we take $a=n, b=n, c=n$ and $d=n$, we obtain

$$
Q(n, n, n, n)=F_{n+1}^{3} F_{n}(1+i+j+k) .
$$

We can designate it as

$$
\begin{aligned}
Q(n, 0,0,0) & =F_{n}, \quad Q(0, n+1,0,0)=i F_{n+1}, \\
Q(0,0, n+2,0) & =j F_{n+2}, \quad Q(0,0,0, n+3)=k F_{n+3} .
\end{aligned}
$$

By using the relations above, we reach the Fibonacci quaternion

$$
\begin{aligned}
Q_{n} & =Q(n, 0,0,0)+Q(0, n+1,0,0)+Q(0,0, n+2,0)+Q(0,0,0, n+3) \\
& =F_{n}+i F_{n+1}+j F_{n+2}+k F_{n+3} .
\end{aligned}
$$




\section{Main results}

In this section, it will be indicated how golden-like ratio, and some identities for the Fibonacci quaternion sequence with quadruple-produce components (14) are obtained. The generating and the exponential generating functions, and its series and binomial sum formula will be derived.

We can state the golden-like ratio for the Fibonacci quaternion sequence with quadruple-produce components. We obtain golden-like ratios of the Fibonacci quaternion sequence with quadruple-produce components below using (3):

$$
\lim _{a, b, c, d \rightarrow \infty} \frac{Q(a, b, c, d)}{F_{d} F_{c} F_{b} F_{a}}=\phi^{3}(1+i+j+k),
$$

and likewise,

$$
\lim _{a, b, c, d \rightarrow \infty} \frac{Q(a, b, c, d)}{F_{d+1} F_{c+1} F_{b+1} F_{a+1}}=\frac{1}{\phi}(1+i+j+k)
$$

or

$$
\lim _{n \rightarrow \infty} \frac{Q(n+1, n+1, n+1, n+1)}{Q(n, n, n, n)}=\phi^{4} .
$$

Proposition 1. If $a, b, c, d$ are positive integers, then
A. $Q(a, b, c, d)+\overline{Q(a, b, c, d)}=2 F_{d+1} F_{c+1} F_{b+1} F_{a}$,
B. $Q(a, b, c, d) \overline{Q(a, b, c, d)}=F_{d+1}^{2} F_{c+1}^{2} F_{b+1}^{2} F_{a}^{2}+F_{d+1}^{2} F_{c+1}^{2} F_{b}^{2} F_{a+1}^{2}$

$$
+F_{d+1}^{2} F_{c}^{2} F_{b+1}^{2} F_{a+1}^{2}+F_{d}^{2} F_{c+1}^{2} F_{b+1}^{2} F_{a+1}^{2},
$$
C. $Q(a, b, c, d) Q(a, b, c, d)=-F_{d+1}^{2} F_{c+1}^{2} F_{b+1}^{2} F_{a}^{2}-F_{d+1}^{2} F_{c+1}^{2} F_{b}^{2} F_{a+1}^{2}$
$-F_{d+1}^{2} F_{c}^{2} F_{b+1}^{2} F_{a+1}^{2}-F_{d}^{2} F_{c+1}^{2} F_{b+1}^{2} F_{a+1}^{2}+2 F_{d+1} F_{c+1} F_{b+1} F_{a} Q(a, b, c, d)$,
D. $Q(a, b, c, d)^{2}+Q(a, b, c, d) \overline{Q(a, b, c, d)}=2 F_{d+1} F_{c+1} F_{b+1} F_{a} Q(a, b, c, d)$.

Proof. The claims A and B are obvious because of the properties (14) and (15) of quaternions, and the claims $\mathrm{C}$ and $\mathrm{D}$ follow by easy calculations.

Theorem 1. The Fibonacci sum, for the Fibonacci quaternion sequence with quadruple-produce components, is

$$
G_{S}=\sum_{d, c, b, a=0}^{m} Q(a, b, c, d)=(1+i+j+k)\left(F_{m+3}-1\right)^{3}\left(F_{m+2}-1\right) .
$$

Proof. Using (14) and (5), we write

$$
G_{S}=\sum_{d, c, b, a=0}^{m} F_{d+1} F_{c+1} F_{b+1} F_{a}+i \sum_{d, c, b, a=0}^{m} F_{d+1} F_{c+1} F_{b} F_{a+1}
$$




$$
\begin{aligned}
& +j \sum_{d, c, b, a=0}^{m} F_{d+1} F_{c} F_{b+1} F_{a+1}+k \sum_{d, c, b, a=0}^{m} F_{d} F_{c+1} F_{b+1} F_{a+1} \\
= & \sum_{d, c, b=0}^{m} F_{d+1} F_{c+1} F_{b+1}\left(F_{m+2}-1\right)+i \sum_{d, c, b=0}^{m} F_{d+1} F_{c+1} F_{b}\left(F_{m+3}-1\right) \\
& +j \sum_{d, c, b=0}^{m} F_{d+1} F_{c} F_{b+1}\left(F_{m+3}-1\right)+k \sum_{d, c, b=0}^{m} F_{d} F_{c+1} F_{b+1}\left(F_{m+3}-1\right) \\
= & \sum_{d, c=0}^{m} F_{d+1} F_{c+1}\left(F_{m+3}-1\right)\left(F_{m+2}-1\right)+i \sum_{d, c=0}^{\infty} F_{d+1} F_{c+1}\left(F_{m+3}-1\right)\left(F_{m+2}-1\right) \\
& +\left(j \sum_{d, c=0}^{m} F_{d+1} F_{c}+k \sum_{d, c=0}^{m} F_{d} F_{c+1}\right)\left(F_{m+3}-1\right)^{2} \\
= & \left(\sum_{d=0}^{m} F_{d+1}+i \sum_{d=0}^{m} F_{d+1}\right)\left(F_{m+3}-1\right)^{2}\left(F_{m+2}-1\right) \\
& +j \sum_{d=0}^{m} F_{d+1}\left(F_{m+3}-1\right)^{2}\left(F_{m+2}-1\right)+k \sum_{d=0}^{m} F_{d}\left(F_{m+3}-1\right)^{3} \\
= & (1+i+j+k)\left(F_{m+3}-1\right)^{3}\left(F_{m+2}-1\right) .
\end{aligned}
$$

Theorem 2. The generating function, for the Fibonacci quaternion sequence with quadruple-produce components, is

$$
G_{Q}(x)=\sum_{d, c, b, a=0}^{\infty} Q(a, b, c, d) x^{a+b+c+d}=(1+i+j+k) \frac{x}{\left(1-x-x^{2}\right)^{4}} .
$$

Proof. Using (14) and (6), we write

$$
\begin{aligned}
& G_{Q}(x)=\sum_{d, c, b, a=0}^{\infty} F_{d+1} F_{c+1} F_{b+1} F_{a} x^{a+b+c+d}+i \sum_{d, c, b, a=0}^{\infty} F_{d+1} F_{c+1} F_{b} F_{a+1} x^{a+b+c+d} \\
& +j \sum_{d, c, b, a=0}^{\infty} F_{d+1} F_{c} F_{b+1} F_{a+1} x^{a+b+c+d}+k \sum_{d, c, b, a=0}^{\infty} F_{d} F_{c+1} F_{b+1} F_{a+1} x^{a+b+c+d} \\
& =\sum_{d, c, b=0}^{\infty} F_{d+1} F_{c+1} F_{b+1} x^{b+c+d}\left(\frac{x}{1-x-x^{2}}\right) \\
& \quad+i \sum_{d, c, b=0}^{\infty} F_{d+1} F_{c+1} F_{b} x^{b+c+d}\left(\frac{1}{1-x-x^{2}}\right)
\end{aligned}
$$




$$
\begin{aligned}
& +j \sum_{d, c, b=0}^{\infty} F_{d+1} F_{c} F_{b+1} x^{b+c+d}\left(\frac{1}{1-x-x^{2}}\right) \\
& +k \sum_{d, c, b=0}^{\infty} F_{d} F_{c+1} F_{b+1} x^{b+c+d}\left(\frac{1}{1-x-x^{2}}\right) \\
& =\sum_{d, c=0}^{\infty} F_{d+1} F_{c+1} x^{c+d}\left(\frac{1}{1-x-x^{2}}\right)\left(\frac{x}{1-x-x^{2}}\right) \\
& +i \sum_{d, c=0}^{\infty} F_{d+1} F_{c+1} x^{c+d}\left(\frac{1}{1-x-x^{2}}\right)\left(\frac{x}{1-x-x^{2}}\right) \\
& +j \sum_{d, c=0}^{\infty} F_{d+1} F_{c} x^{c+d}\left(\frac{1}{1-x-x^{2}}\right)^{2} \\
& +k \sum_{d, c=0}^{\infty} F_{d} F_{c+1} x^{c+d}\left(\frac{1}{1-x-x^{2}}\right)^{2} \\
& =\sum_{d=0}^{\infty} F_{d+1} x^{d}\left(\frac{1}{1-x-x^{2}}\right)^{2}\left(\frac{x}{1-x-x^{2}}\right) \\
& +i \sum_{d=0}^{\infty} F_{d+1} x^{d}\left(\frac{1}{1-x-x^{2}}\right)^{2}\left(\frac{x}{1-x-x^{2}}\right) \\
& +j \sum_{d=0}^{\infty} F_{d+1} x^{d}\left(\frac{1}{1-x-x^{2}}\right)^{2}\left(\frac{x}{1-x-x^{2}}\right) \\
& +k \sum_{d=0}^{\infty} F_{d} x^{d}\left(\frac{1}{1-x-x^{2}}\right)^{3} \\
& =(1+i+j+k) \frac{x}{\left(1-x-x^{2}\right)^{4}} \text {. }
\end{aligned}
$$

Theorem 3. The exponential generating function, for the Fibonacci quaternion sequence with quadruple-produce components, is

$$
\begin{aligned}
E_{Q}(x) & =\sum_{d, c, b, a=0}^{\infty} \frac{Q(a, b, c, d)}{a ! b ! c ! d !} x^{a+b+c+d} \\
& =(1+i+j+k)\left(\frac{e^{\alpha x}-e^{\beta x}}{\alpha-\beta}\right)\left(\frac{\alpha e^{\alpha x}-\beta e^{\beta x}}{\alpha-\beta}\right)^{3},
\end{aligned}
$$

where $\alpha$ and $\beta$ are defined by (2). 
Proof. Using (14) and (7), we write

$$
\begin{aligned}
& E_{Q}(x)=\sum_{d, c, b, a=0}^{\infty} \frac{F_{d+1} F_{c+1} F_{b+1} F_{a}}{a ! b ! c ! d !} x^{a+b+c+d}+i \sum_{d, c, b, a=0}^{\infty} \frac{F_{d+1} F_{c+1} F_{b} F_{a+1}}{a ! b ! c ! d !} x^{a+b+c+d} \\
& +j \sum_{d, c, b, a=0}^{\infty} \frac{F_{d+1} F_{c} F_{b+1} F_{a+1}}{a ! b ! c ! d !} x^{a+b+c+d}+k \sum_{d, c, b, a=0}^{\infty} \frac{F_{d} F_{c+1} F_{b+1} F_{a+1}}{a ! b ! c ! d !} x^{a+b+c+d} \\
& =\sum_{d, c, b=0}^{\infty} \frac{F_{d+1} F_{c+1} F_{b+1}}{b ! c ! d !} x^{b+c+d}\left(\frac{e^{\alpha x}-e^{\beta x}}{\alpha-\beta}\right) \\
& +i \sum_{d, c, b=0}^{\infty} \frac{F_{d+1} F_{c+1} F_{b}}{b ! c ! d !} x^{b+c+d}\left(\frac{\alpha e^{\alpha x}-\beta e^{\beta x}}{\alpha-\beta}\right) \\
& +j \sum_{d, c, b=0}^{\infty} \frac{F_{d+1} F_{c} F_{b+1}}{b ! c ! d !} x^{b+c+d}\left(\frac{\alpha e^{\alpha x}-\beta e^{\beta x}}{\alpha-\beta}\right) \\
& +k \sum_{d, c, b=0}^{\infty} \frac{F_{d} F_{c+1} F_{b+1}}{b ! c ! d !} x^{b+c+d}\left(\frac{\alpha e^{\alpha x}-\beta e^{\beta x}}{\alpha-\beta}\right) \\
& =\sum_{d, c=0}^{\infty} \frac{F_{d+1} F_{c+1}}{c ! d !} x^{c+d}\left(\frac{e^{\alpha x}-e^{\beta x}}{\alpha-\beta}\right)\left(\frac{\alpha e^{\alpha x}-\beta e^{\beta x}}{\alpha-\beta}\right) \\
& +i \sum_{d, c=0}^{\infty} \frac{F_{d+1} F_{c+1}}{c ! d !} x^{c+d}\left(\frac{e^{\alpha x}-e^{\beta x}}{\alpha-\beta}\right)\left(\frac{\alpha e^{\alpha x}-\beta e^{\beta x}}{\alpha-\beta}\right) \\
& +j \sum_{d, c=0}^{\infty} \frac{F_{d+1} F_{c}}{c ! d !} x^{c+d}\left(\frac{\alpha e^{\alpha x}-\beta e^{\beta x}}{\alpha-\beta}\right)^{2} \\
& +k \sum_{d, c=0}^{\infty} \frac{F_{d} F_{c+1}}{c ! d !} x^{c+d}\left(\frac{\alpha e^{\alpha x}-\beta e^{\beta x}}{\alpha-\beta}\right)^{2} \\
& =\sum_{d=0}^{\infty} \frac{F_{d+1}}{d !} x^{d}\left(\frac{e^{\alpha x}-e^{\beta x}}{\alpha-\beta}\right)\left(\frac{\alpha e^{\alpha x}-\beta e^{\beta x}}{\alpha-\beta}\right)^{2} \\
& +i \sum_{d=0}^{\infty} \frac{F_{d+1}}{d !} x^{d}\left(\frac{e^{\alpha x}-e^{\beta x}}{\alpha-\beta}\right)\left(\frac{\alpha e^{\alpha x}-\beta e^{\beta x}}{\alpha-\beta}\right)^{2} \\
& +j \sum_{d=0}^{\infty} \frac{F_{d+1}}{d !} x^{d}\left(\frac{e^{\alpha x}-e^{\beta x}}{\alpha-\beta}\right)\left(\frac{\alpha e^{\alpha x}-\beta e^{\beta x}}{\alpha-\beta}\right)^{2} \\
& +k \sum_{d=0}^{\infty} \frac{F_{d}}{d !} x^{d}\left(\frac{\alpha e^{\alpha x}-\beta e^{\beta x}}{\alpha-\beta}\right)^{3}
\end{aligned}
$$




$$
=(1+i+j+k)\left(\frac{e^{\alpha x}-e^{\beta x}}{\alpha-\beta}\right)\left(\frac{\alpha e^{\alpha x}-\beta e^{\beta x}}{\alpha-\beta}\right)^{3} .
$$

Theorem 4. The series of the Fibonacci quaternion sequence with quadruple-produce components is

$$
F_{s}=\sum_{d, c, b, a=0}^{\infty} \frac{Q(a, b, c, d)}{t^{a+b+c+d+4}}=\frac{t^{3}}{\left(t^{2}-t-1\right)^{4}}(1+i+j+k) .
$$

Proof. Using (14) and (8), we write

$$
\begin{aligned}
F_{s}= & \sum_{d, c, b, a=0}^{\infty} \frac{F_{d+1} F_{c+1} F_{b+1} F_{a}}{t^{a+b+c+d+4}}+i \sum_{d, c, b, a=0}^{\infty} \frac{F_{d+1} F_{c+1} F_{b} F_{a+1}}{t^{a+b+c+d+4}} \\
& +j \sum_{d, c, b, a=0}^{\infty} \frac{F_{d+1} F_{c} F_{b+1} F_{a+1}}{t^{a+b+c+d+4}}+k \sum_{d, c, b, a=0}^{\infty} \frac{F_{d} F_{c+1} F_{b+1} F_{a+1}}{t^{a+b+c+d+4}} \\
= & \sum_{d, c, b=0}^{\infty} \frac{F_{d+1} F_{c+1} F_{b+1}}{t^{b+c+d+3}} \frac{1}{t^{2}-t-1}+i \sum_{d, c, b=0}^{\infty} \frac{F_{d+1} F_{c+1} F_{b}}{t^{b+c+d+2}} \frac{1}{t^{2}-t-1} \\
& +j \sum_{d, c, b=0}^{\infty} \frac{F_{d+1} F_{c} F_{b+1}}{t^{b+c+d+2}} \frac{1}{t^{2}-t-1}+k \sum_{d, c, b=0}^{\infty} \frac{F_{d} F_{c+1} F_{b+1}}{t^{b+c+d+2}} \frac{1}{t^{2}-t-1} \\
= & \sum_{d, c=0}^{\infty} \frac{F_{d+1} F_{c+1}}{t^{c+d+1}} \frac{1}{\left(t^{2}-t-1\right)^{2}}+i \sum_{d, c=0}^{\infty} \frac{F_{d+1} F_{c+1}}{t^{c+d+1}} \frac{1}{\left(t^{2}-t-1\right)^{2}} \\
& +j \sum_{d, c=0}^{\infty} \frac{F_{d+1} F_{c}}{t^{c+d}} \frac{1}{\left(t^{2}-t-1\right)^{2}}+k \sum_{d, c=0}^{\infty} \frac{F_{d} F_{c+1}}{t^{c+d}} \frac{1}{\left(t^{2}-t-1\right)^{2}} \\
= & \sum_{d=0}^{\infty}\left(\frac{t^{3} F_{d+1}}{t^{d+2}}+i \frac{t^{3} F_{d+1}}{t^{d+2}}+j \frac{t^{3} F_{d+1}}{t^{d+2}}+k \frac{t^{3} F_{d}}{t^{d+1}}\right) \frac{1}{\left(t^{2}-t-1\right)^{3}} \\
= & \frac{t^{3}}{\left(t^{2}-t-1\right)^{4}}(1+i+j+k) .
\end{aligned}
$$

The work of [5] provides insight into the proof of the following theorem.

Theorem 5. The binomial sum, for the Fibonacci quaternion sequence with quadruple-produce components, is

$$
F_{B}=\sum_{d, c, b, a=0}^{m}\left(\begin{array}{c}
m \\
d
\end{array}\right)\left(\begin{array}{c}
m \\
c
\end{array}\right)\left(\begin{array}{c}
m \\
b
\end{array}\right)\left(\begin{array}{c}
m \\
a
\end{array}\right) Q(a, b, c, d)=(1+i+j+k) F_{2 m+1}^{3} F_{2 m} .
$$


Proof. Using (14) and (9), we write

$$
\begin{aligned}
& F_{B}=\sum_{d, c, b, a=0}^{m}\left(\begin{array}{c}
m \\
d
\end{array}\right)\left(\begin{array}{c}
m \\
c
\end{array}\right)\left(\begin{array}{c}
m \\
b
\end{array}\right)\left(\begin{array}{l}
m \\
a
\end{array}\right) F_{d+1} F_{c+1} F_{b+1} F_{a} \\
& +i \sum_{d, c, b, a=0}^{m}\left(\begin{array}{c}
m \\
d
\end{array}\right)\left(\begin{array}{c}
m \\
c
\end{array}\right)\left(\begin{array}{c}
m \\
b
\end{array}\right)\left(\begin{array}{c}
m \\
a
\end{array}\right) F_{d+1} F_{c+1} F_{b} F_{a+1} \\
& +j \sum_{d, c, b, a=0}^{m}\left(\begin{array}{l}
m \\
d
\end{array}\right)\left(\begin{array}{c}
m \\
c
\end{array}\right)\left(\begin{array}{c}
m \\
b
\end{array}\right)\left(\begin{array}{c}
m \\
a
\end{array}\right) F_{d+1} F_{c} F_{b+1} F_{a+1} \\
& +k \sum_{d, c, b, a=0}^{m}\left(\begin{array}{l}
m \\
d
\end{array}\right)\left(\begin{array}{c}
m \\
c
\end{array}\right)\left(\begin{array}{c}
m \\
b
\end{array}\right)\left(\begin{array}{l}
m \\
a
\end{array}\right) F_{d} F_{c+1} F_{b+1} F_{a+1} \\
& =\sum_{d, c, b=0}^{m}\left(\begin{array}{l}
m \\
d
\end{array}\right)\left(\begin{array}{c}
m \\
c
\end{array}\right)\left(\begin{array}{c}
m \\
b
\end{array}\right) F_{d+1} F_{c+1} F_{b+1} F_{2 m} \\
& +i \sum_{d, c, b=0}^{m}\left(\begin{array}{c}
m \\
d
\end{array}\right)\left(\begin{array}{c}
m \\
c
\end{array}\right)\left(\begin{array}{c}
m \\
b
\end{array}\right) F_{d+1} F_{c+1} F_{b} F_{2 m+1} \\
& +j \sum_{d, c, b=0}^{m}\left(\begin{array}{c}
m \\
d
\end{array}\right)\left(\begin{array}{c}
m \\
c
\end{array}\right)\left(\begin{array}{c}
m \\
b
\end{array}\right) F_{d+1} F_{c} F_{b+1} F_{2 m+1} \\
& +k \sum_{d, c, b=0}^{m}\left(\begin{array}{c}
m \\
d
\end{array}\right)\left(\begin{array}{c}
m \\
c
\end{array}\right)\left(\begin{array}{c}
m \\
b
\end{array}\right) F_{d} F_{c+1} F_{b+1} F_{2 m+1} \\
& =\sum_{d, c=0}^{m}\left(\begin{array}{l}
m \\
d
\end{array}\right)\left(\begin{array}{c}
m \\
c
\end{array}\right) F_{d+1} F_{c+1} F_{2 m+1} F_{2 m}+i \sum_{d, c=0}^{m}\left(\begin{array}{c}
m \\
d
\end{array}\right)\left(\begin{array}{c}
m \\
c
\end{array}\right) F_{d+1} F_{c+1} F_{2 m+1} F_{2 m} \\
& +j \sum_{d, c=0}^{m}\left(\begin{array}{l}
m \\
d
\end{array}\right)\left(\begin{array}{c}
m \\
c
\end{array}\right) F_{d+1} F_{c} F_{2 m+1}^{2}+k \sum_{d, c=0}^{m}\left(\begin{array}{c}
m \\
d
\end{array}\right)\left(\begin{array}{c}
m \\
c
\end{array}\right) F_{d} F_{c+1} F_{2 m+1}^{2} \\
& =\sum_{d=0}^{m}\left(\begin{array}{c}
m \\
d
\end{array}\right) F_{d+1} F_{2 m+1}^{2} F_{2 m}+i \sum_{d=0}^{m}\left(\begin{array}{c}
m \\
d
\end{array}\right) F_{d+1} F_{2 m+1}^{2} F_{2 m} \\
& +j \sum_{d=0}^{m}\left(\begin{array}{c}
m \\
d
\end{array}\right) F_{d+1} F_{2 m+1}^{2} F_{2 m}+k \sum_{d=0}^{m}\left(\begin{array}{l}
m \\
d
\end{array}\right) F_{d} F_{2 m+1}^{3} \\
& =(1+i+j+k) F_{2 m+1}^{3} F_{2 m} \text {. }
\end{aligned}
$$

Acknowledgements. The authors would like to thank the anonymous referee for useful comments and advices. 


\section{References}

[1] Y. Alagoz, K. H. Oral, and S. Yucel, Split quaternion matrices, Math. Notes (Miskolc) 13(2) (2012), 223-232.

[2] G. Bilgici, U. Tokeser, and Z. Unal, Fibonacci and Lucas sedenions, J. Integer Seq. 20(1) (2017), Article 17.1.8, 11 pp.

[3] C. Cimen and A. İpek, On pell quaternions and Pell-Lucas quaternions, Adv. Appl. Clifford Algebr. 26(1) (2016), 39-51.

[4] G. B. Djordevic and H. M. Srivastava, Some generalizations of the incomplete Fibonacci and the incomplete Lucas polynomials, Adv. Stud. Contemp. Math. (Kyungshang) 11(1) (2005), 11-32.

[5] G. B. Djordevic and H. M. Srivastava, Some generalizations of certain sequences associated with the Fibonacci numbers, J. Indones. Math. Soc. 12 (2006), 99-112.

[6] S. Halici, On Fibonacci quaternions, Adv. Appl. Clifford Algebr. 22(2) (2012), 321327.

[7] C. J. Harman, Complex Fibonacci numbers, Fibonacci Quart. 19(1) (1981), 82-86.

[8] A. F. Horadam, Complex Fibonacci numbers and Fibonacci quaternions, Amer. Math. Monthly 70 (1963), 289-291.

[9] A. F. Horadam and S. Pethe, Euclidean coordinates as generalized Fibonacci numbers products, Fibonacci Quart. 24(4) (1986), 366-370.

[10] A. F. Horadam, Quaternion recurrence relations, Ulam Quart. 2(2) (1993), 23-33.

[11] A. Ipek, On $(p, q)$-Fibonacci quaternions and their Binet formulas, generating functions and certain binomial sums, Adv. Appl. Clifford Algebr. 27(2) (2017), 1343-1351.

[12] T. Koshy, Fibonacci and Lucas Numbers with Applications. Volume 1, John Wiley \& Sons, New Jersey, 2018.

[13] M. Shafiq, H. M. Srivastava, N. Khan, Q. Z. Ahmad, M. Darus, and S. Kiran, An upper bound of the third Hankel determinant for a subclass of q-starlike functions associated with k-Fibonacci numbers, Symmetry 12(6) (2020), 1043.

[14] H. M. Srivastava, Operators of basic (or $q_{-}^{-}$) calculus and fractional $q$-calculus and their applications in geometric function theory of complex analysis, Iran. J. Sci. Technol. Trans. A Sci. 44(1) (2020), 327-344.

[15] H. M. Srivastava, N. Tuglu, and M. Cetin, Some results on the q-analogues of the incomplete Fibonacci and Lucas polynomials, Math. Notes (Miskolc) 20(1) (2019), $511-524$.

[16] D. Tasci and F. Yalcin, Complex Fibonacci p-numbers, Commun. Math. Appl. 4(3) (2013), 213-218.

[17] F. Zhang, Quaternions and matrices of quaternions, Linear Algebra Appl. 251 (1997), 21-57.

Mersin University, Graduate School of Natural and Applied Sciences, 33343 Mersin, Turkey

E-mail address: orhandiskaya@mersin.edu.tr

Mersin University, Faculty of Arts and Science, Dept. of Mathematics, 33343 Mersin, Turkey

E-mail address: hmenken@mersin.edu.tr

URL: http://apbs.mersin.edu.tr/hmenken 